\title{
Glut 1, S-100, and Nerve Bundle Study in Vascular Anomalies
}

\author{
Ajay K. Khanna ${ }^{1} \cdot$ Akhilesh Kumar $^{1} \cdot$ Soumya Khanna ${ }^{2} \cdot$ Amrita Kar $^{3} \cdot$ Puneet Kumar $^{1} \cdot$ S. K. Tiwary ${ }^{1}$
}

Received: 13 May 2021 / Accepted: 24 May 2021 / Published online: 18 June 2021

(c) The Author(s) 2021

\begin{abstract}
Vascular anomalies grouped into vascular tumors (hemangioma) (HI) and vascular malformation (VM) are benign vascular lesions that are difficult to distinguish from one another clinically and often confused with each other at histopathology. This confusing terminology leads to improper diagnosis, illogical treatment, and misdirected research. This study aimed to study GLUT 1, S-100, and nerve bundle to differentiate hemangioma and vascular malformation. Thirty two cases of vascular lesions (26 vascular malformations and 6 hemangiomas) were taken into the study. For histological evaluation and immunohistochemistry (IHC), samples of vascular lesions were collected in formalin. All the hematoxylin and eosin-stained slides were evaluated under light microscope for histology and nerve bundles. Immunohistochemical staining was performed by streptavidin-biotin method for GLUT 1 and S-100. GLUT 1 was positive in all 6 cases of hemangiomas (100\%) and only in 1 case of vascular malformation. Nerve bundle was present in 24 cases of vascular malformation (92.3\%) out of 26 cases but not in any cases of hemangioma and S-100 was found positive in all vascular malformation cases (100\%) but not in hemangioma. So GLUT 1 expression, S-100, and presence of nerve bundle in vascular lesions can help to differentiate hemangioma and vascular malformation.
\end{abstract}

Keywords Pathology $\cdot$ Malformation $\cdot$ Venous $\cdot$ Arterial $\cdot$ Capillary $\cdot$ IHC

\section{Introduction}

Vascular anomalies grouped as vascular malformation (VM) and hemangiomas (Hi) are benign vascular lesions that are difficult to distinguish from one another clinically and often confused with each other at histopathology. This confusing terminology leads to improper diagnosis, illogical treatment, and misdirected research.

Vascular malformations are the result of errors in morphogenesis and are divided into subtypes based on the constituent vessels: capillary, venous, arterial, lymphatic, and combined forms. They are a complex network of intercommunicating arterial and venous structures. Hemangiomas, on the other hand, result from a derangement in angiogenesis

Soumya Khanna

khannaasoumya15@gmail.com

1 Department of General Surgery, Institute of Medical Sciences, Banaras Hindu University, Varanasi 221005, India

2 Department of Anatomy, Institute of Medical Sciences, Banaras Hindu University, Varanasi 221005, India

3 Department of Pathology, Institute of Medical Sciences, Banaras Hindu University, Varanasi 221005, India with exuberant proliferation of vascular elements due to imbalance between angiogenic and angiostatic forces. Therefore, arteries and arterioles are not part of the lesion.

The diagnosis and pathogenesis of these lesions continue to challenge histopathologists, who are often called on to help with the definitive diagnosis. The presence of arteries, arterioles, or both as an integral part of the lesions is often used as a diagnostic criterion for differentiating vascular malformations from hemangiomas. Also, the presence of intralesional nerve in vascular malformation provides an additional diagnostic criterion that is simple and reliable and can be readily used to differentiate vascular malformation from hemangiomas; even in H\&E-stained tissue sections or S-100 for nerve and nerve fibers, it has been used. Specific immunohistochemical markers such as erythrocyte-type glucose transporter protein 1 (GLUT 1) and S-100 have been described to differentiate hemangiomas from vascular malformations. This study aimed to use GLUT 1, S-100, and nerve bundle proposed to differentiate between hemangioma and vascular malformation. 


\section{Patients and Methods}

This study has been carried out in the Department of General Surgery and Department of Pathology at Banaras Hindu University, India, from July 2018 to July 2020. Out of 32 lesions, 6 were hemangiomas, and 26 were vascular malformation, and a detailed study of clinical parameters, histological diagnosis, GLUT 1 staining, S-100 staining, and nerve fibers was carried out. This case series study was approved by the Institute's ethical committee. Only surface hemangiomas and vascular malformations were included in the study. The patients with varicose vein, trauma-associated AVM, and intracranial lesion and visceral hemangiomas were excluded.

Tissue samples were collected following surgical procedure. For histological evaluation and immunohistochemistry (IHC), 32 samples of vascular lesions were collected in formalin. All the hematoxylin and eosin-stained slides of selected cases were evaluated under light microscope for histology especially for nerve fibers.

Immunohistochemical staining was performed by streptavidin-biotin method using a LSAB kit (Dako, Denmark) for GLUT 1 (Neomarkers, CA, USA, 1:100) and S-100 protein (Zymed, CA, USA, 1:500). Slides were visualized under light microscope. GLUT 1 expression in endothelial cells and S-100 expression in nerve bundle were noted. Expression of GLUT 1 in endothelial cells was reported as positive, and absence of GLUT 1 expression was reported as negative. Similarly, expression of S-100 in nerve bundle was reported as positive, and absence of S-100 was reported as negative.

The statistical analysis was done using the SPSS for Windows version 23.0 software (IBM Inc.). For categorical data, Chi-square and Fischer's exact test were used. For comparing two groups of mean, independent Student's 't' test was used. The critical value of 'p' indicating the probability of significant difference was taken as $<0.05$ for comparison.

\section{Results}

This study was carried out on 32 patients (26 vascular malformations and 6 hemangiomas). The clinical details are shown in Table 1. Eight patients were less than 15 years; 19 were men. All hemangiomas were present at birth, and 20 out of 26 vascular malformations were noted at birth. Majority of the patients had the lesion for more than 12 months (Table 2). Majority of the hemangiomas were in the neck while majority of vascular malformations were in the limbs (Table 3).

In $26(100 \%)$ cases of vascular malformation, S-100 is positive, and none of the hemangioma showed S-100
Table 1 General parameters

\begin{tabular}{lll}
\hline Parameter & Hemangioma (6) & $\begin{array}{l}\text { Vascular } \\
\text { malformation } \\
(26)\end{array}$ \\
\hline Age $<15$ years & $3(50 \%)$ & $5(19.2 \%)$ \\
Age $>15$ years & $3(50 \%)$ & $21(80.8 \%)$ \\
Men & $5(83.3 \%)$ & $14(53.8 \%)$ \\
Women & $1(16.7 \%)$ & $12(46.2 \%)$ \\
Present at birth & $4(66.6 \%)$ & $20(76.9 \%)$ \\
\hline
\end{tabular}

positivity with $\mathrm{p}$ value of $<0.001$ that is significant. This indicates that $S-100$ shows positivity mainly in vascular malformation (Table 4, Fig. 1). GLUT 1 is positive in all 6 cases of hemangiomas $(100 \%)$ and in 1 case of vascular malformation (3.8\%) with $\mathrm{p}$ value of 0.001 which is significant, suggesting GLUT 1 shows positivity mainly in hemangioma (Fig. 2). Nerve bundle is present mainly in venous malformations and none of the case of hemangioma with $p$ value of $<0.001$ that is statically significant (Fig. 3).

The sensitivity of GLUT 1 for hemangiomas was $100 \%$ with specificity of $96.15 \%$, positive predictive value of $85.71 \%$ and negative predictive value of $100 \%$, and diagnostic accuracy of $96.88 \%$, while the sensitivity of GLUT 1 for vascular malformations was $3.84 \%$, with specificity of $0 \%$, positive predictive value of $14.29 \%$, negative predictive value of $0 \%$, and diagnostic accuracy of $3.12 \%$. The sensitivity, specificity, positive predictive value, negative predictive value, and diagnostic accuracy of S-100 were $100 \%$ for vascular malformation and zero for hemangiomas. The sensitivity of nerve bundles for hemangiomas was $0 \%$ with specificity of $7.69 \%$, positive predictive value of $0 \%$, negative predictive value of $25 \%$, and diagnostic accuracy of $6.24 \%$, while the sensitivity of nerve bundle for vascular malformations was $92.31 \%$, with specificity of $100 \%$, positive predictive value of $100 \%$, negative predictive value of $75 \%$, and diagnostic accuracy of $93.75 \%$.

\section{Discussion}

The current classification system of vascular anomalies is based on the landmark investigation published in 1982, based on biological activity [1]. The International Society for the Study of Vascular Anomalies adopted this classification

Table 2 Duration of lesion

\begin{tabular}{lll}
\hline & Hemangioma (6) & $\begin{array}{l}\text { Vascular mal- } \\
\text { formations } \\
(26)\end{array}$ \\
\hline Less than 6 months & 0 & $4(15.4 \%)$ \\
6-12 months & $1(6.7 \%)$ & $3(11.5 \%)$ \\
More than 12 months & $5(83.3 \%)$ & $19(73.1 \%)$ \\
\hline
\end{tabular}


Table 3 Distribution according to site

\begin{tabular}{llllll}
\hline Site & \multicolumn{2}{l}{ Hemangioma } & & \multicolumn{2}{l}{$\begin{array}{l}\text { Vascular malfor- } \\
\text { mation }\end{array}$} \\
\cline { 2 - 3 } \cline { 5 - 6 } & No & & & No & $\%$ \\
\hline Rt. upper limb & 0 & 0 & 7 & 26.9 \\
Rt. lower limb & 1 & 16.7 & & 3 & 11.5 \\
Lt. upper limb & 0 & 0 & & 4 & 15.4 \\
Lt. lower limb & 2 & 33.3 & 6 & 23.1 \\
Head & 0 & 0 & & 2 & 7.7 \\
Neck & 3 & 50 & 3 & 11.5 \\
Back & 0 & 0 & & 1 & 3.8 \\
Total & 6 & 100 & 26 & 100 \\
\hline
\end{tabular}

for vascular anomalies in 1996 (Fig. 4). Further, this classification system was recently expanded at the 2014 ISSVA workshop in Melbourne. This revision again provides much greater detail including newly named anomalies and identified genes to account for recent advances in knowledge and clinical associations [2]. As a result, we now recognize 2 main types of vascular anomalies: vascular tumors and vascular malformations. Differentiating between these 2 types is essential because their treatment is quite different. The management of vascular anomalies is a dynamic and rapidly developing subspecialty, which requires interdisciplinary collaboration and multidisciplinary intervention. Vascular tumors have been differentiated from vascular malformations based on their clinical appearance, radiological and pathological features, and biological behavior (Table 5).

Hemangiomas tend to be small or absent at birth and often are not initially noticed. Shortly after birth, they undergo a proliferative phase, with rapid growth that may last several months. They then undergo a stationary period, followed by a period of involution. Conversely, vascular malformations are always present at birth and enlarge in proportion to the growth of the child. They do not involute and remain present throughout the patient's life. Vascular malformations are subcategorized as lymphatic, capillary, venous, arteriovenous, and mixed malformations on the basis of their histologic makeup. The diagnosis and pathogenesis of these lesions continue to challenge histopathologists [3]. Majority of the vascular malformations are slow flow of which more than half comprised of venous malformations, and the rest was formed by capillary, lymphatic, combined slow flow, and fast flow malformations [4]. Further, they are mainly

Table 4 Positive distribution of GLUT 1, S-100, and nerve bundle in $\mathrm{Hi}$ and VM

\begin{tabular}{llll}
\hline & GLUT 1 & Nerve bundle & S-100 \\
\hline Hemangioma (6) & $6(100 \%)$ & 0 & 0 \\
Vascular malformation (26) & $1(3.8 \%)$ & $24(92.3 \%)$ & $26(100 \%)$ \\
\hline
\end{tabular}

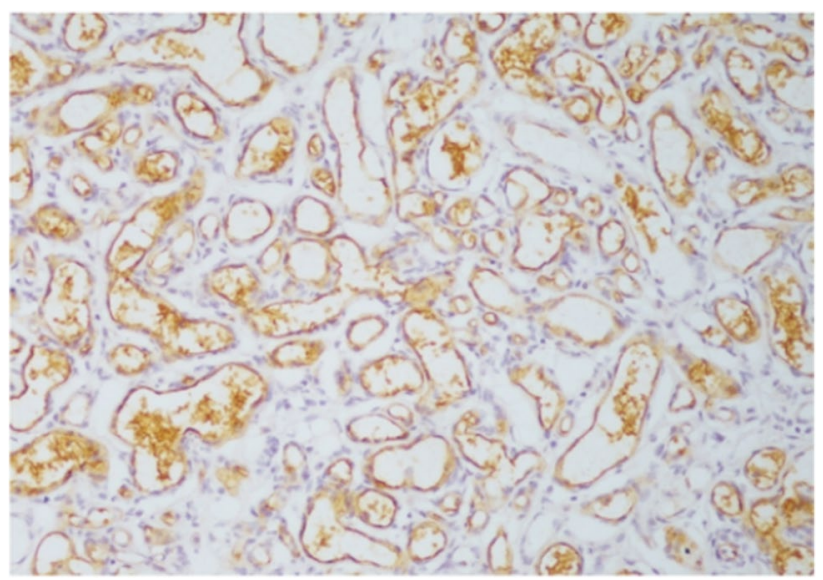

Fig. 1 Glut-1 positivity

venous in origin [5], and these lesions are mainly in cephalic area in $70 \%$, upper limbs in $17 \%$, and lower limb involvement in $11 \%$ [6]. In another study, lower extremity formed the majority $42 \%$ of the total cases, followed by hemicorporal $32 \%$, upper extremity $10 \%$, and head and neck $3 \%$ was least in venous malformations [7].

Specific immunohistochemical markers such as erythrocyte-type glucose transporter protein 1 (GLUT 1) have been described to differentiate hemangiomas from vascular malformations. GLUT 1 (glucose transporter isoform 1) is an immunohistochemical marker that is normally restricted to the endothelial cells that have a blood-tissue barrier function, such as those in the brain and placenta. Specific microvascular GLUT 1 (erythrocyte-type glucose transporter protein) immunoreactivity is present in normal brain cells, placental chorionic villi, and placental trophoblasts, but this is undetectable in the vasculature of normal skin and the subcutis layer. GLUT 1 to be highly expressed in the majority of lesional vessels of late involuting - as well as of early proliferative-phase

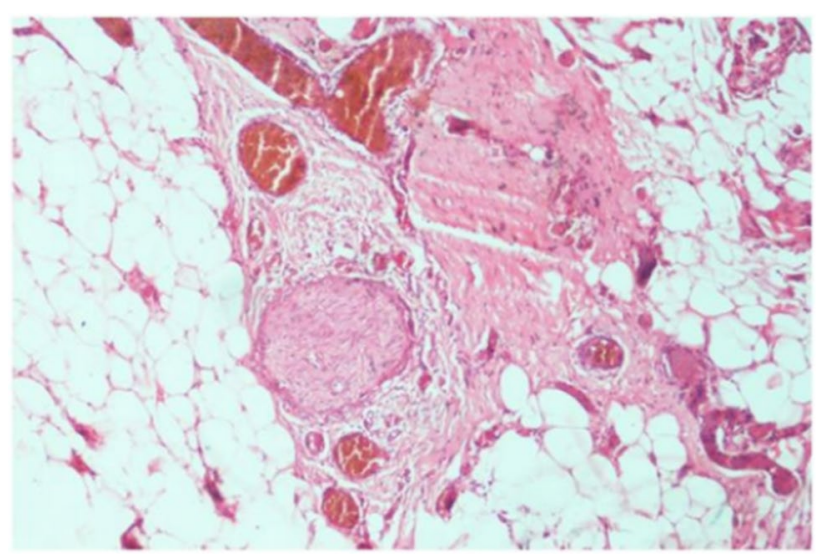

Fig. 2 Nerve bundles 


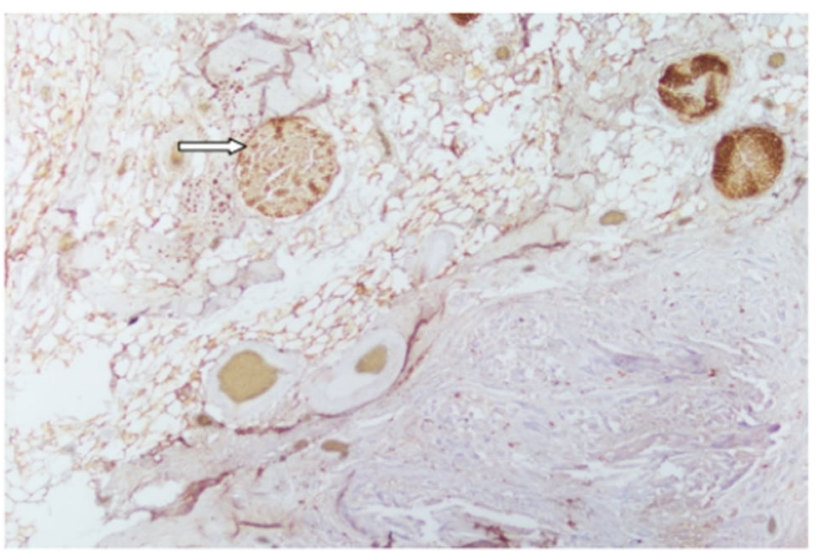

Fig. 3 S-100 immunostaining

hemangiomas. Indicating that GLUT 1 expression in these tumors is independent of mitotic activity. Similarly, other studies suggest GLUT 1 to be a reliable and highly specific immunohistochemical marker for hemangiomas $[8,9]$.

A study of orbital vascular anomalies analyzed ten cases of $\mathrm{HI}$ and ten cases of orbital encapsulated cavernous venous lesions or orbital VMs. They found that all cases of HI were positive for GLUT 1, whereas none of the VM cases were positive [10]. Further, in another study, immunohistochemical expression of 30 cases initially diagnosed as "hemangiomas" and 30 cases diagnosed as oral pyogenic granuloma (PG) were studied. The immunohistochemical test showed that only seven of the "hemangioma" cases were true HIs. Specimens that were GLUT 1 negative were reclassified as oral PG (ten) or VM. None of the PG cases were positive for GLUT 1, and so their initial histological diagnoses were maintained. In view of these findings, the authors concluded that histopathological characteristics are not alone enough to guarantee correct diagnosis of oral HIs [11]. In our study, GLUT 1 expression was found in all 6 cases of hemangioma, and one case of vascular malformation and 25 cases of vascular malformation did not express GLUT 1. GLUT 1 in diagnosis of hemangioma had sensitivity of $100 \%$, specificity of $96.15 \%$, and positive predictive value of $85.71 \%$.

The presence of intralesional nerve in AVM provides an additional diagnostic criterion that is simple and reliable and can be readily used to differentiate AVMs from hemangiomas; even in H\&E-stained tissue sections or S-100, an immunohistochemical stain for nerve and nerve fibers is used. Only a handful of previous studies have focused on the presence or distribution of nerves in benign vascular lesions. Rydh et al. [12]. reported absence of nerve bundles and paucity of nerve fibers around the dilated vessels in 9 cases of port-wine stains (which they called venous malformations) and concluded that loss of vascular tone due to absence of adequate nerve supply may be responsible for the vascular ectasia that characterizes those lesions. Considering the absence of nerve bundles in those lesions, we suggest they are better classified as venous hemangiomas. Another study of 167 cases found that nerve bundles are consistently present in vascular malformations and absent in hemangiomas and so can be used as a diagnostic clue to differentiate between these lesions [13]. In our study, nerve bundle was absent in all cases of hemangioma and 2 cases of vascular malformation but present in $24(92.3 \%)$ in vascular malformation.

S-100 is an immunostain used to detect nerve fiber in vascular lesion. S-100 derives its name from the fact that it is soluble in saturated (100\%) ammonium sulphate. In a
Fig. 4 Classification of vascular anomalies. (Adapted from the International Society for the Study of Vascular Anomalies) AM arterial malformation, AVF arteriovenous fistula, AVM arteriovenous malformation, CLAVM capillary-lymphaticarteriovenous, VM venous malformation, LM lymphatic malformation, CM capillary malformation, CLVM capillary lymphatic venous malformation, VL venous lymphatic malformation

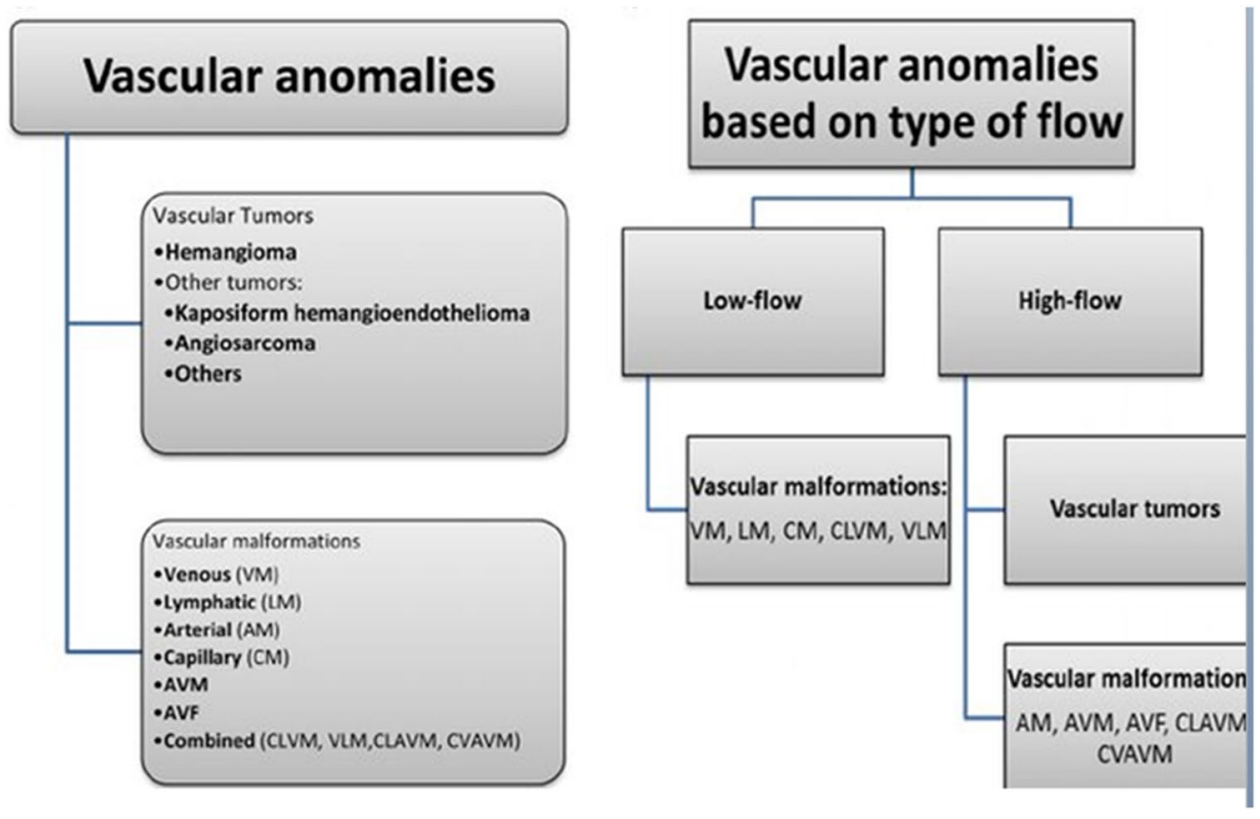


Table 5 Differentiating features between hemangioma and vascular malformations

\begin{tabular}{|c|c|c|}
\hline & Hemangioma & Vascular malformation \\
\hline Biological nature & Vascular tumors (proliferative) & Non-proliferative \\
\hline Age at presentation & Birth/infancy & Variable, may present at birth or early adulthood \\
\hline Categories & Focal/segmental, superficial/deep & Arterial/venous/capillary, Truncal/extra-truncal \\
\hline Clinical appearance & Discolored/pinkish nodules, plaques, patches & $\begin{array}{l}\text { Superficial lesions as cluster of vessels, pulsations may be } \\
\text { visible with AVM, bluish discolored veins soft compress- } \\
\text { ible in venous malformations, deep-seated lesion present as } \\
\text { audible bruits or as venous edema of extremity }\end{array}$ \\
\hline Natural history & Proliferative and involution phases & $\begin{array}{l}\text { Tend to grow proportionately with age, disproportionate } \\
\text { growth at pregnancy/adolescence, rarely involute }\end{array}$ \\
\hline Duplex findings & $\begin{array}{l}\text { Proliferative: High flow } \\
\text { Involution: Low flow }\end{array}$ & High/low flow depending on arteriovenous/venous/lymphatic \\
\hline MR angiography & $\begin{array}{l}\text { Proliferative: low signal intensity lobulated mass on } \mathrm{T} 1 \text { and } \\
\text { high intensity on T2-weighted images, flow voids with } \\
\text { T1 SE } \\
\text { Involution: high signal intensity masses on T1 }\end{array}$ & $\begin{array}{l}\text { AVM: demarcated feeding vessels and abnormal venous } \\
\text { return } \\
\text { Venous malformation: low signal intensity in T1-weighted } \\
\text { images, high intensity on T2-weighted images and flow } \\
\text { voids in T2-weighted fat-saturated images }\end{array}$ \\
\hline
\end{tabular}

study of 6 intramuscular hemangiomas using S-100 immunohistochemical stain. They reported the nerve content of hemangiomas to be the same as that in normal tissue and that in surrounding margins of the lesions. They also observed increased presence of nerves in the immediate (1-3 mm) vicinity of the intramuscular hemangiomas [14]. In our study of 32 patients, S-100 was found positive in all 26 cases of vascular malformation and negative in hemangioma. Nerve bundle was present in 24 cases of vascular malformation and absent in all cases of hemangioma.

Vascular malformations encompass a wide range of diseases often associated with somatic or, more rarely, germinal genetic mutations. A mutation in the PIK3Ca/mTOR pathway is more often involved in various VMs. CD10 and CD34 are cellular markers that may play a role in mesenchymal differentiation and proliferation [15]. The first treatment to inhibit this pathway with sirolimus indicated that molecular treatment can be effective against VMs. In addition, certain VM "hotspot" mutations have been previously found in tumors, providing the rationale for the exploration and repurposing of existing and investigational cancer drugs for VMs. Finally, discoveries of molecular and cellular abnormalities that characterize a large proportion of VMs and the generation of pre-clinical VM mouse models provide the necessary basis for the development of the targeted molecular treatment strategies [16].

We propose that the protocol for differentiating hemangiomas from vascular malformations is to look for nerve bundle on histology. If nerve bundles are seen, then, that is suggestive of vascular malformation rather than hemangiomas. Further, S-100 immunostaining can be done again to see for nerve bundle. To confirm it as hemangioma, GLUT 1 should be done. If it comes out to be positive, then, it should be taken as hemangioma.

\section{Conclusion}

It is sometimes very difficult to differentiate between hemangioma and vascular malformation. Certain parameters as GLUT 1 expression, S-100, and presence of nerve bundle can differentiate between these two conditions. Glut 1 expression was present in all 6 cases of hemangioma and one case of vascular malformation, and 25 cases of vascular malformation did not express GLUT 1. Nerve bundle was absent in all cases of hemangioma and 2 cases of vascular malformation but present in 24 (92.3\%) in vascular malformation. S-100 was found positive in $26(100 \%)$ in vascular malformation and absent in all 6 (100\%) in hemangioma. So GLUT 1 is almost diagnostic of hemangioma while S-100 and nerve fiber diagnostic of vascular malformation.

\section{Declarations}

Conflict of Interest The authors declare no competing interest.

Open Access This article is licensed under a Creative Commons Attribution 4.0 International License, which permits use, sharing, adaptation, distribution and reproduction in any medium or format, as long as you give appropriate credit to the original author(s) and the source, provide a link to the Creative Commons licence, and indicate if changes were made. The images or other third party material in this article are included in the article's Creative Commons licence, unless indicated otherwise in a credit line to the material. If material is not included in the article's Creative Commons licence and your intended use is not permitted by statutory regulation or exceeds the permitted use, you will need to obtain permission directly from the copyright holder. To view a copy of this licence, visit http://creativecommons.org/licenses/by/4.0/. 


\section{References}

1. Mulliken JB, Glowacki J (1982) Hemangiomas and vascular malformations in infants and children: a classification based on endothelial characteristics. Plast Reconstr Surg 69:412-422

2. Dasgupta R, Fishman SJ (2014) ISSVA classification. Semin Pediatr Surg 23(4):158-161

3. Wójcickia P, Wójcickaaf K (2014) Epidemiology, diagnostics and treatment of vascular tumours and malformations. Adv Clin Exp Med 23(3):475-484

4. Upton J, Coombs CJ, Mulliken JB, Burrows PE, Pap S (1999) Vascular malformations of the upper limb: a review of 270 patients. J Hand Surg Am 24(5):1019-1035

5. Redondo P, Bastarrika G, Aguado L, Martínez-Cuesta A, Sierra A, Cabrera J, Alonso-Burgos A (2009) Foot or hand malformations related to deep venous system anomalies of the lower limb in Klippel-Trénaunay syndrome. J Am Acad Dermatol 61(4):621-628

6. Enjolras O, Mulliken JB (1997) Vascular tumors and vascular malformations (new issues). Adv Dermatol 13:375-423

7. North PE, Waner M, Mizeracki A, Mihm MC Jr (2000) GLUT 1: a newly discovered immunohistochemical marker for juvenile hemangiomas. Hum Pathol 31(1):11-22

8. van Vugt LJ, van der Vleuten CJM, Flucke U, Blokx WAM (2017) The utility of GLUT 1 as a diagnostic marker in cutaneous vascular anomalies: a review of literature and recommendations for daily practice. Pathol Res Pract 213(6):591-597

9. Johann AC, Salla JT, Gomez RS, Aguiar MC, Gontijo B, Mesquita RA (2007) GLUT-1 in oral benign vascular lesions. Oral Dis 13(1):51-55

10. Osaki TH, Jakobiec FA, Mendoza PR, Lee Y, Fay AM (2013) Immunohistochemical investigations of orbital infantile hemangiomas and adult encapsulated cavernous venous lesions (malformation versus hemangioma). Ophthal Plast Reconstr Surg 29(3):183-195

11. Oliveira DH, Silveira EJ, Medeiros AM, Alves PM, Queiroz LM (2014) Study of the etiopathogenesis and differential diagnosis of oral vascular lesions by immunoexpression of GLUT-1 and HIF-1 $\alpha$. J Oral Pathol Med 43(1):76-80

12. Rydh M, Malm M, Jernbeck J, Dalsgaard CJ (1991) Ectatic blood vessels in port-wine stains lack innervations: possible role in pathogenesis. Plast Reconstr Surg 87:419-422

13. Patrick A (2005) Adegboyega, Suimin Qiu. Hemangioma versus vascular malformation presence of nerve bundle is a diagnostic clue for vascular malformation, Arch Pathol Lab Med 129:772-775

14. Robinson D, Segal M, Halperin N, Nevo Z (1992) Neuropeptidergic innervations of intramuscular hemangiomas. Exp Mol Pathol 56:186-196

15. Moneghini L, Tosi D, Graziani D, Caretti A, Colletti G, Baraldini V, Cattaneo E, Spaccini L, Zocca A, Bulfamante GP (2020) CD10 and CD34 as markers in vascular malformations with PIK3CA and TEK mutations. Hum Pathol 99:98-106

16. Kangas J, Nätynki M, Eklund L (2018) Development of molecular therapies for venous malformations. Basic Clin Pharmacol Toxicol 123(Suppl 5):6-19

Publisher's Note Springer Nature remains neutral with regard to jurisdictional claims in published maps and institutional affiliations. 